Journal of Machine Engineering, 2021, Vol. 21, No. 3, 92-100

ISSN 1895-7595 (Print) ISSN 2391-8071 (Online)

Received: 19 April 2021 / Accepted: 18 June 2021 / Published online: 19 July 2021

wire-arc additive manufacturing, thermal indicator, thermal online monitoring, high temperature zone

\author{
Matthieu RAUCH ${ }^{1,2 *}$ \\ Jean-Yves HASCOET ${ }^{1,2}$ \\ Clement ROUSSEAU ${ }^{1,2}$ \\ Guillaume RUCKERT ${ }^{2,3}$
}

\title{
THERMAL MONITORING FOR METALLIC ADDITIVE MANUFACTURING MULTI-BEADS MULTI-LAYERS PARTS
}

\begin{abstract}
Among Metallic Additive Manufacturing processes, Directed Energy Deposition (DED) processes are very promising for the Industry. An issue that prevents a larger development of DED is the reliability of the process, since its complexity makes the result of the manufacturing variable. Thermal behavior is a critical aspect for which uncontrolled phenomena can lead to part failure. Some thermal monitoring and closed-loop control methods have been developed, that enables to observe and regulate the heating of the processed part. However, these methods rely on local measures from a region or a single external surface of a part, and thus provide partial information of thermal fields in the whole part volume. This paper proposes a method that combines diverse data to compute online a process indicator that is meaningful for the thermal state of the whole part, and hence for the control of the manufacturing of multi-beads multi-layer parts. A simulation-based model using thermal partial data is proposed. An online monitoring experiment is proposed for validation of the model. Relevance of the control method to ensure mechanical properties of the part is then tested.
\end{abstract}

\section{INTRODUCTION}

Metallic additive manufacturing has developed over the pasts years, and is more and more used by the industry as the control on the processes is improved, and the costs are reduced.

Among those processes, Directed Energy Deposition (DED) are not the most common for now, but provides several advantages. DED processes are Metallic Additive Manufacturing processes whose particularity is to bring in the same spot the energy input and the matter input that are needed for the deposition [1]. This enables the manufacturing of large parts, or a greater material deposition rate [2], with a good buy-to-fly ratio [3].

\footnotetext{
${ }^{1}$ GeM - UMR CNRS 6183, Centrale Nantes, France

${ }^{2}$ Additive Manufacturing Group, Joint Laboratory of Marine Technology (JLMT) Centrale Nantes - Naval Group, France

${ }^{3}$ Naval Group Research, Technocampus Ocean, France

*E-mail: Matthieu.Rauch@ec-nantes.fr https://doi.org/10.36897/jme/139158
} 
The WAAM (Wire-Arc Additive Manufacturing) process uses arc welding to deposit beads in successive layers, and thus manufacture a part that can have a relatively complex shape [4].

However, several issues prevent the development of DED technologies in the Industry. Due to the complexity of the process, manufacturing reliability is not ensured, and a proper set of manufacturing parameters must be found for each part. Several failure cases cannot easily be modelled and simulated, and will need development of specialized modelling tools as well as further research concerning the needed numerical methods [5]. Therefore, trial-anderror is the most used approach to determinate an appropriate set of parameters, what is costly for prototypes and small series [6].

One of the source of defects or failure of the parts is the risk of heat accumulation in the part over time, especially for large part. Overheating can cause bead collapse, excessive thermomechanical residual stress, or bad microstructure of deposited material [7]. Thus, it is a major challenge for research to propose approaches for the control of thermal behaviour of manufacturing for enhancement of the reliability of DED processes, especially for WAAM that uses the highest deposition rate, energy input, and part size.

Several methodologies have been investigated to prevent heat accumulation. Some of them use active cooling systems [8]. It has the downside of adding another system to the machine. Thus, other methodologies focus on adapting the process to prevent heat accumulation. Process parameters can be changed, and depositing strategies can be adapted. Simulations are used to predict thermal and mechanical state of the part through the process, and hence find suitable parameters [6]. Nevertheless, the complexity of the process makes the simulation process complex and costly also [9], as the simulation of the behaviour of the melt pool at a micro scale [10]. Macro scale simulations may be easier to conduct but remains less precise to predict failure.

Real-time monitoring can be implemented to verify the validity of a part during the deposition, and acknowledge the appearance of defects while recording information that enables a better comprehension of the underlying physics of the process [9]. Figure 1 shows the concept of closed-loop control of DED processes. The first step to achieve closed-loop control is to set up a proper monitoring device. Then, it is necessary to process monitored raw data into relevant indicators linked with the physics of the process and with the result of the manufacturing. Finally, it is possible to use indicators as inputs of a controller that will adapt process parameters to achieve control of the process.

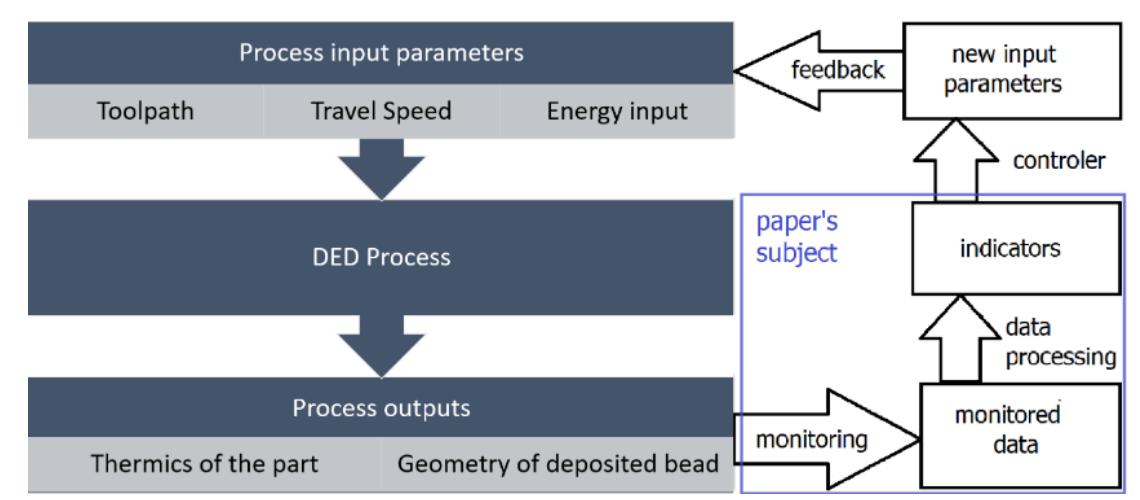

Fig. 1. Context of the study 
Several authors have introduced thermal monitoring. Some of the monitoring methodologies focus on the local aspect of the process, like monitoring of the size and temperature of the molten pool [11]. These approach provides indicators that are very close to the process physics, but have also present a lack of information concerning the global thermal aspect of the part, since thermal accumulation has effects on the melting pool but happens in the whole part.

Hence, other approaches achieve monitoring of temperature fields at the scale of the part on a side of the part with paraxial infrared (IR) thermography [12]. This way, a lot of information is acquired and various indicators can be computed. A widely used indicator of heat accumulation at part's scale is the size of a High Temperature Zone (HTZ), defined as the surface whose temperature exceeds a defined temperature [13]. More complex indicators based can be used to predict and control the material's mechanical properties as hardness [14].

One of the limits of this approach is that the observed information used to determine the part's thermal behaviour is limited to the temperature on a single external surface. Therefore, this approach is unsuitable for multi bead parts like thick parts or complex shape parts.

The approach proposed in this paper is to use simulation data to develop a simple model that enables the computation of thermal indicators that are relevant for the deposition of multibead thick parts, with only the IR thermography of an external surface of the manufactured part as an input.

First, manufacturing of thick parts with WAAM process is carried out along with simulations of those processes. Simulation are adjusted to fit with monitored data from the real processes.

Thereafter is discussed the relevance of several thermal indicators for multi-beads parts. Thermal analysis is led to construct simplified models dedicated to calculation of those thermal indicators, given the temperature fields and the thickness of the part. The accuracy of those models is verified with the analysis of the microstructure of the deposited parts.

Eventually, the computation of thermal indicators is implemented in the monitoring device in order to monitor these indicators in real-time. Tests are led to verify the pertinence of the indicators given the properties of deposited part. Further possible applications of developed methodology are then discussed.

\section{EXPERIMENTS AND SIMULATIONS}

\subsection{PROCESSING TEST PARTS}

Two test parts are manufactured with the WAAM process. The purpose of the experiment is to monitor the thermal fields of parts during deposition, to identify properly the material's thermal parameters for simulation. The Gas Metal Arc Welding (GMAW) equipment is placed on a KUKA KR500 6-axis articulated robot. The process used is Cold Metal Transfer (CMT) welding. The substrate was preheated to $250^{\circ} \mathrm{C}$ before the beginning of the deposition. 
The manufactured parts are nominally rectangular cuboids made of duplex stainless steel. The trajectories are $100 \mathrm{~mm}$ long beads deposited back and forth. Every layer is similar to the previous, the torch position shifted up by the height of a layer. Figure 2 shows pictures of the part after deposition. The difference between the two parts is the number of beads in each layer, respectively 5 in part A and 9 in part B. In Table 1 are indicated the containing dimensions of the two manufactured parts.

Table 1. Measured dimensions of manufactured parts

\begin{tabular}{|l|c|c|}
\cline { 2 - 3 } \multicolumn{1}{c|}{} & Part A & Part B \\
\hline Height $(\mathrm{mm})$ & 70.7 & 81.2 \\
\hline Length $(\mathrm{mm})$ & 106.2 & 105.3 \\
\hline Width $(\mathrm{mm})$ & 18.9 & 27.5 \\
\hline
\end{tabular}
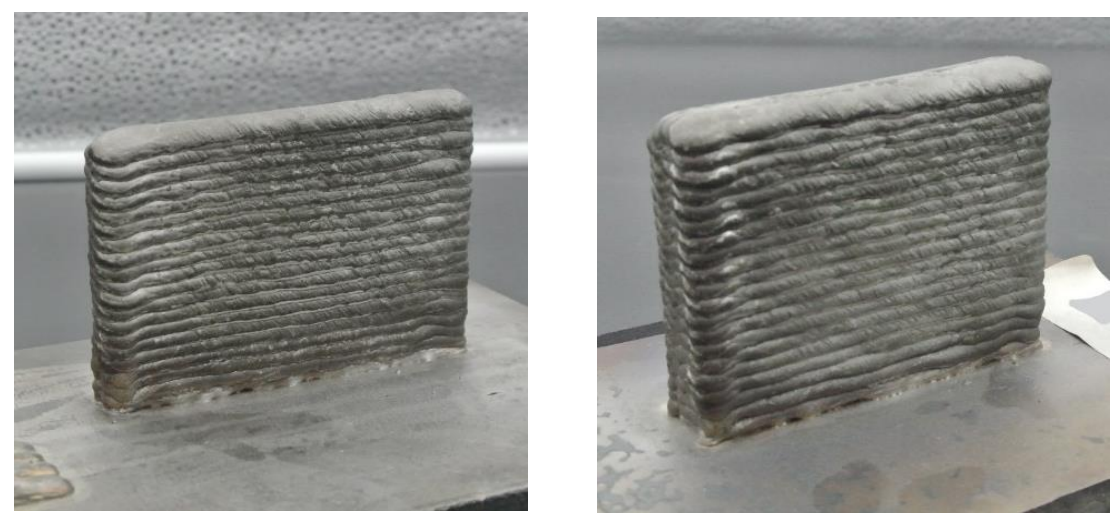

Fig. 2. Manufactured parts A (left) and B (right)

The process is monitored with an infrared camera, placed 1 meter away from the middle of deposited part. This camera capabilities are detailed in Table 2.

Table 2. Capabilities of the infrared camera

\begin{tabular}{|l|c|}
\hline \multicolumn{1}{|c|}{ Camera range $\left({ }^{\circ} \mathrm{C}\right)$} & $300^{\circ} \mathrm{C}-2000^{\circ} \mathrm{C}$ \\
\hline Maximal Image frequency $(\mathrm{Hz})$ & 12 \\
\hline Thermal sensivity at ambient temperature $(\mathrm{mK})$ & 50 \\
\hline Spatial resolution $(\mathrm{mrad} / \mathrm{pixel})$ & 0.41 \\
\hline
\end{tabular}

The calibration of the camera is done in accordance with results from previous work with the same conditions [15]. Temperature fields are displayed and recorded in real time as greyscale images. Figure 3 shows a greyscale image of the deposition of part B, where grey levels corresponds linearly to temperatures. This monitoring methodology enables online processing of thermal fields and computation of various parameters.

Thus, thermal gradients and cooling rates are computed for every frame and every pixel. This data can be treated for local analysis as well as for the computation of global indicators. 


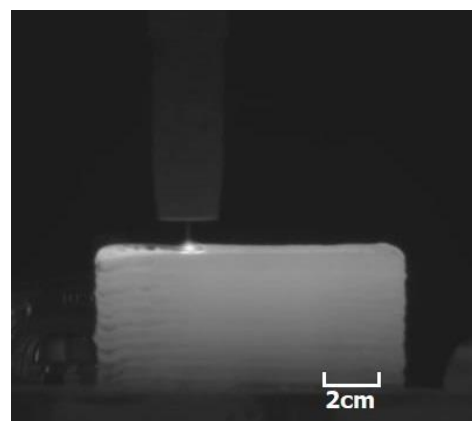

Fig. 3. Thermal image of the deposition of part B

High temperature zones are computed for different temperatures. HTZ are defined as zones in the thermal image where temperature exceeds a predefined value. HTZ can be a proper indicator of heat accumulation during the deposition of a part [16]. HTZs can be visualized during manufacturing, as shown in Fig. 4, as well as indicators like HTZs' sizes.

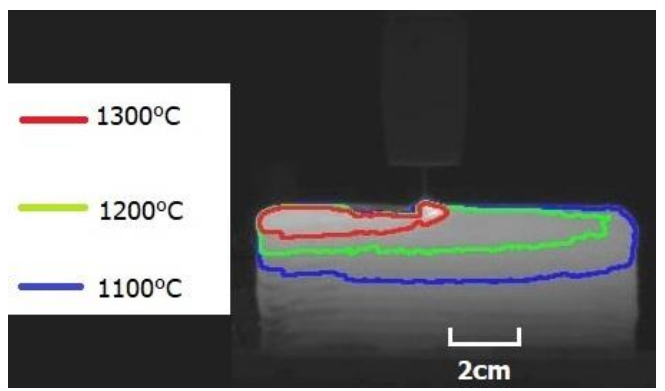

Fig. 4. HTZ detection for part A

Figure 5 shows, through the evolution of HTZs size, the accumulation of heat in part A during deposition. However, a lack of information is noted as for the comparison between the two parts, since these parts are considered only for one single external surface.

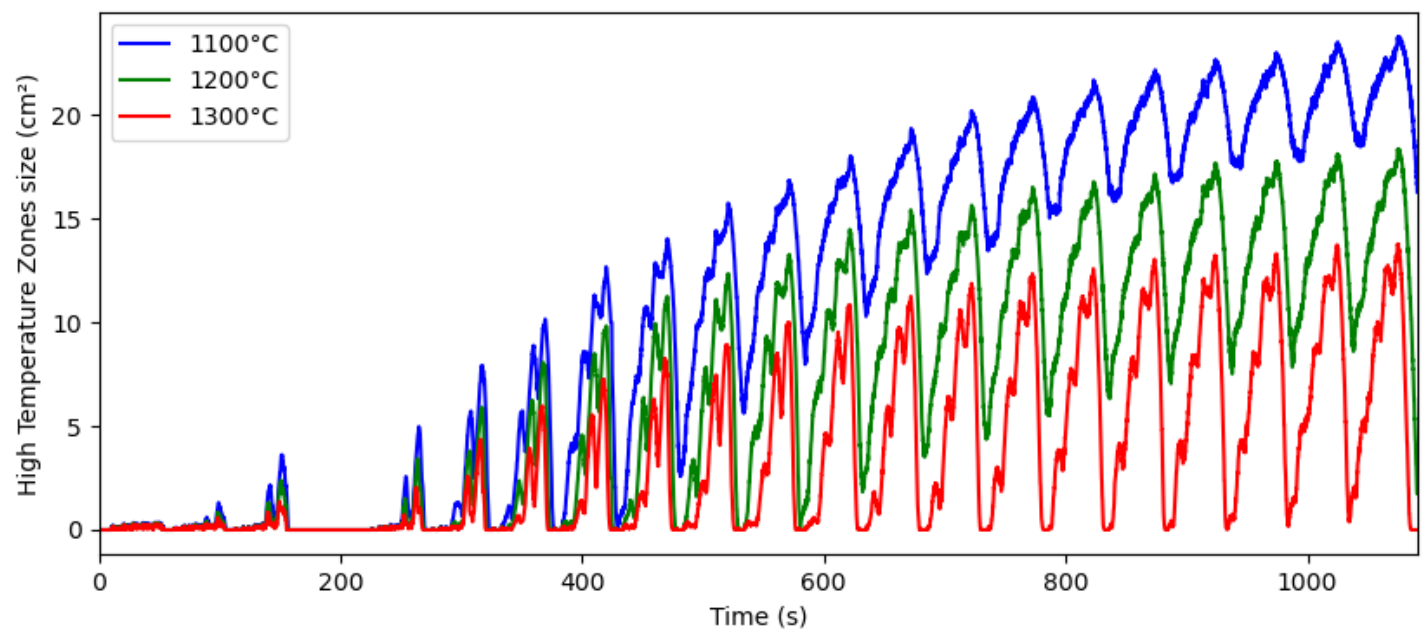

Fig. 5. HTZ sizes evolution for part A 
Simulation of the processing of these two parts is led with a simulation software, following the simulation method developed in [9]. The simulation is designed to correspond to the real process results and not the nominal part. Figure 6 represents the results of a simulation.

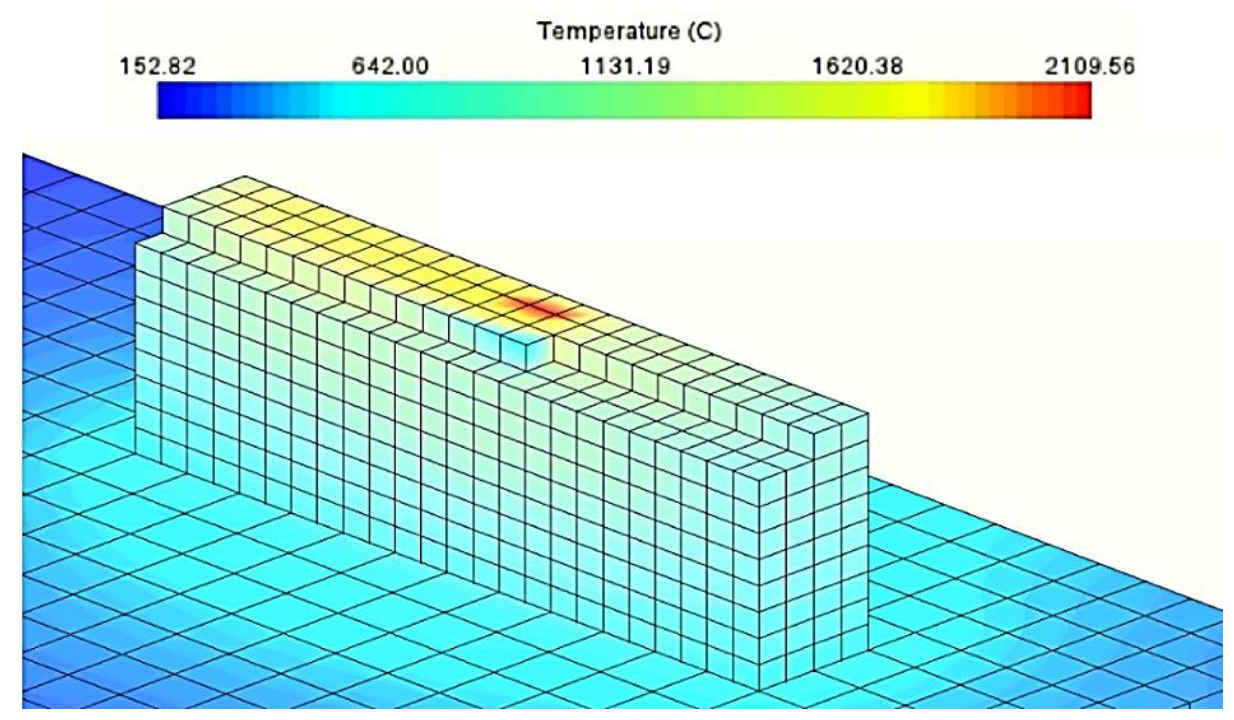

Fig. 6. Simulation of part A's deposition

Thermal data that is used for ensuring compliance of the simulation are temperature fields and temperature gradients on the external surface that coincide with monitored surface on real manufactured part. The calibration is done with a simple criteria that compares the temperature of isolated points in the simulation and with thermography data, starting from peak temperature time. The temperature gap is computed and integrated through time. A gradient method is used to converge to the most complying set of parameters, varying input power and convection coefficient, other material parameters being assumed known for the material.

A set of nine points is chosen for this purpose, since it ensure that the temperature are not just correct for one point, but are compared through the two dimensions of the observed surface. Nine points instead of three enables to ensure the correct depiction of temperature fields when the evolution through one direction or another is not linear. The grid of chosen control points is depicted in Fig. 7.

The compared temperature through times of monitored deposited part A and corresponding simulation is depicted in Fig. 8. The simulated temperature in orange has the same behavior and shape than the monitored temperature. Nevertheless, there remains temperature discrepancies that are under a hundred degrees, especially for the upper points of the parts. This differential is due partly to a temporal delay, the simulation having a few seconds delay at the end of the process. Due to the temperature gradient, the gap will be slighter with indicators like High Temperature Zones instead of temperatures of precise points. 


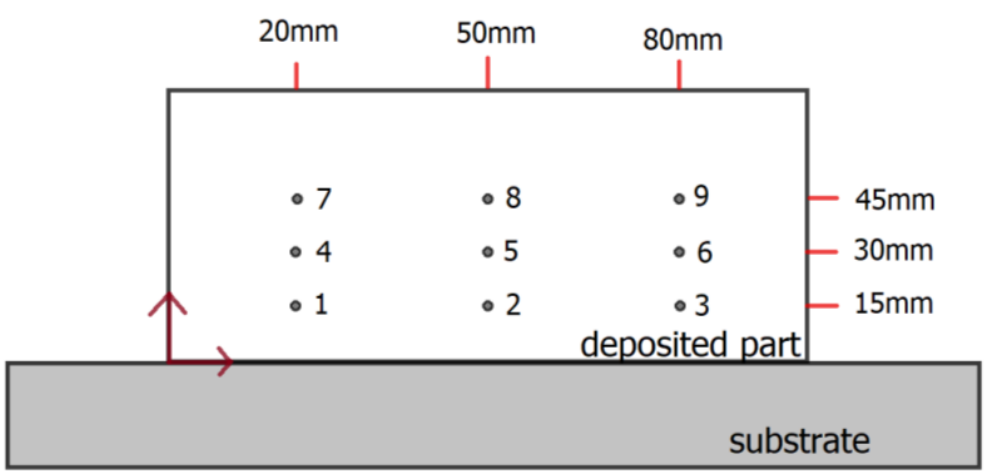

Fig. 7. Control points on the deposited part
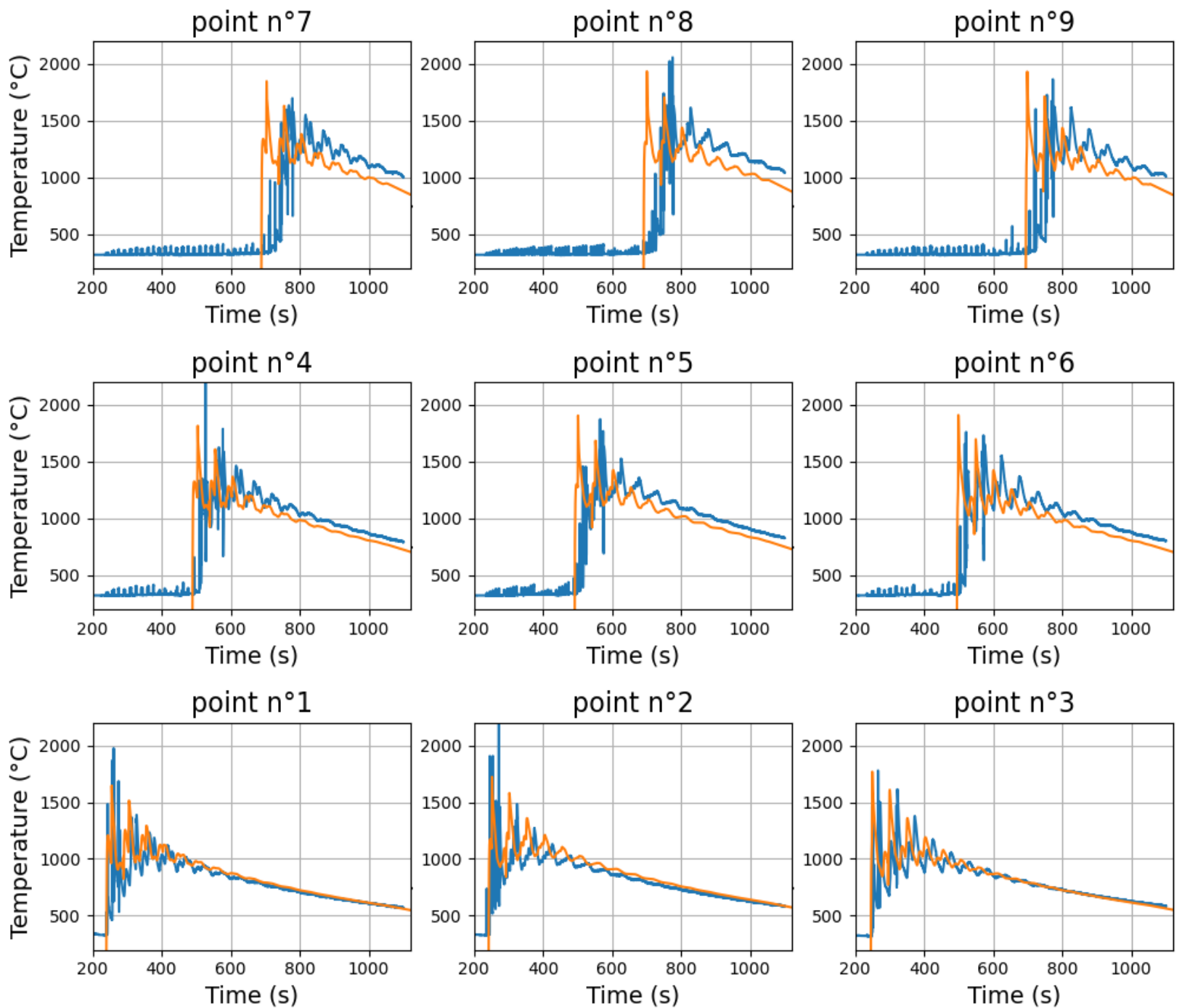

Fig. 8. Compared temperatures $\left({ }^{\circ} \mathrm{C}\right.$ ) of simulated deposition (orange) and deposition monitoring (blue) through time for nine points of the observed surface for part A

After validation, thermal fields from the whole part are used to determinate relations between thermal indicators in the whole part and thermal field on a single external surface. 


\section{THREE-DIMENSIONAL HIGH TEMPERATURE ZONE}

Though multiple thermal indicators can be computed, two indicators have been studied. First, a three-dimensional High Temperature Zone (3DHTZ) size indicator is considered.

In [15] is motivated the interest for HTZ indicators. It proved to be a simple and efficient way to control heat accumulation for DED processes, since temperature at a precise point is not needed. However, it is limited, as well as other geometric indicators such as the size of the molten pool, by the lack of direct relation with the material health at the end of the process.

Moreover, multi-bead parts imply that it is not possible to monitor the temperature of the whole part with a single camera. The deposition of the bead is not always at foreground. With the test parts, result and simulation show that the size of a two-dimensional HTZ on the monitored surface will decrease when the torch deposits beads behind the forefront bead, since the centre of the HTZ is farther.

Hence, it is necessary to filter the size of the computation of two-dimensional HTZ through time in order to link it to the size of a three-dimensional HTZ. Assuming that the size and shape of the HTZ variates slightly during the deposition of a layer, it can be postulated that an estimation of the three-dimensional HTZ can be calculated by multiplying the thickness of the part by the mean value of the size of the two-dimensional HTZ throughout a cycle (that corresponds to the deposition of a layer). This can be obtained by a moving average or a bandpass filter, what both require knowing an estimation of the duration of the deposition of a layer.

Second order models are also investigated and are compared, taking surface HTZ for different temperatures and the three dimensions of the part as entrance parameters and a single volumetric HTZ as output parameter. Simulations are carried out, varying the dimensions of the part to identify the models.

\section{CONCLUSION}

To conclude, thermal online monitoring of WAAM-manufactured parts has been performed. It has been possible to simulate the deposition of several parts and make it correspond to the real monitored processes. A method to monitor in real time thermal behaviour of thick part has been proposed.

A perspective for this work is to adapt this method for other DED processes such as Laser Metal Deposition. It is also desirable to generalize this approach with other types of multi-bead parts, with complex shapes, for which the deposition path does not imply fused beads in a layer. For those, prior simulation will be preponderant over thermal analysis. Eventually, the use of these indicators as controlled parameters of controllers would be beneficial for the improvement of DED processes reliability.

It will also be possible to implement thermal closed-loop control based on the developed indicators. Several process parameters can be adapted, including feed rate, energy input intensity, or temporisation between layers. Thus, the process can be fully controlled on thermal aspects. 


\section{REFERENCES}

1. DING D., PAN Z., CUIURI D., LI H., 2015, Wire-Feed Additive Manufacturing of Metal Components: Technologies, Developments and Future Interests, Int. J. Adv. Manuf. Technol., 81, 465-481.

2. KERNINON J., MOGNOL P., HASCOET J.-Y., LEGONIDEC C., 2008, Effect of Path Strategies on Metallic Parts Manufactured by Additive Process, Solid Freeform Fabrication Symposium, 352-361.

3. VENTURINI G., MONTEVECCHI F., SCIPPA A., CAMPATELLI G., 2016, Optimization of WAAM Deposition Patterns for T-crossing Features, Procedia CIRP, 55, 95-100.

4. BANDYOPADHYAY A., TRAXEL K. D., 2018, Invited Review Article: Metal-Additive Manufacturing-Modelling Strategies for Application-Optimized Designs, Additive manufacturing, 22, 758-774.

5. DENLINGER E.R., HEIGEL J.C., MICHALERIS P., PALMER T.A., 2015, Effect of Inter-Layer Dwell Time on Distortion and Residual Stress in Additive Manufacturing of Titanium and Nickel Alloys, Journal of Materials Processing Technology, 215, 123-131.

6. GENG H., LI, J., XIONG J., LIN X., 2017, Optimisation of Interpass Temperature and Heat Input for Wire and Arc Additive Manufacturing 5A06 Aluminium Alloy, Science and Technology of Welding and Joining, 22, 472-483.

7. MAETZ J.Y., 2014, Évolution de la Microstructure d'un Acier Inoxydable Lean Duplex Lors du Vieillissement, PhD thesis, INSA Lyon.

8. WU B., PAN Z., DING D., CUIURI D., LI H., FEI Z., 2018, The Effects of Forced Interpass Cooling on the Material Properties of Wire Arc Additively Manufactured Ti6Al4V alloy, Journal of Materials Processing Technology, 258, 97-105.

9. DING J., et al., 2011, Thermo-Mechanical Analysis of Wire and Arc Additive Layer Manufacturing Process on Large Multi-Layer Parts, Computational Materials Science, 50, 3315-3322.

10. ZHOU X., ZHANG H., WANG G., BAI X., 2017, Three-Dimensional Numerical Simulation of Arc and Metal Transport in Arc Welding Based Additive Manufacturing, Int. J. of Heat and Mass Transfer, 103, 521-537.

11. HU D., KOVACEVIC R., 2003, Sensing, Modeling and Control for laser-Based Additive Manufacturing, International Journal of Machine Tools and Manufacture, 43, 51-60.

12. DONGQING Y., WANG G., ZHANG G., 2012, Thermal Analysis for Single-Pass Multi-Layer GMAW Based Additive Manufacturing Using Infrared Thermography, Journal of Materials Processing Technology, 244, $215-224$.

13. FARSHIDIANFAR M.H., KHAJEPOUR A., GERLICH A., 2016, Real-Time Control of Microstructure in Laser Additive Manufacturing, International Journal of Advanced Manufacturing Technology, 82/5-8, 1173-1186.

14. CHEN Z., GUO X., SHI J., 2020, Hardness Prediction and Verification Based on Key Temperature Features During the Directed Energy Deposition Process, International Journal of Precision Engineering and Manufacturing - Green Technology, 8, 453-469.

15. CHABOT A., RAUCH M., HASCOËT J.-Y., 2019, Towards a Multi-Sensor Monitoring Methodology for AM Metallic Processes, Welding in the World, 63/3, 759-769.

16. KARNATI S., MATTA N., SPARKS T., LIOU F., 2013, Vision-Based Process Monitoring for Laser Metal Deposition Processes, 24th International Solid Freeform Fabrication Symposium, Austin, TX. 